\title{
The interplay of intention maintenance and cue monitoring in younger and older adults' prospective memory
}

\author{
Nicola Ballhausen ${ }^{1}$ - Katharina M. Schnitzspahn ${ }^{2}$ - Sebastian S. Horn ${ }^{3}$. \\ Matthias Kliegel ${ }^{1,4,5}$
}

Published online: 9 June 2017

(C) Psychonomic Society, Inc. 2017

\begin{abstract}
The retention phase of a prospective memory (PM) task poses different challenges, including demands to store or maintain an intended action and to realize the right moment for action execution. The interplay of these processes in younger and older adults has not been explored so far. In this study, the authors examined the impact of maintenance load and task focality on PM in 84 younger and in 83 older adults. Results indicated that PM performance and ongoing task response times were strongly affected by maintenance load and age. However, a focality effect only emerged when maintenance load was low but not when attentional resources were deployed for maintaining a more demanding intention. These findings suggest that maintenance and monitoring requirements compete for similar attentional resources. Furthermore, maintenance load may affect postretrieval processes through its impact on working-memory resources, which can restrain the typical advantage of focal over nonfocal PM tasks.
\end{abstract}

Keywords Prospective memory $\cdot$ Maintenance $\cdot$ Monitoring $\cdot$ Focality $\cdot$ Aging

Nicola Ballhausen

nicola.ballhausen@unige.ch

1 Department of Psychology, University of Geneva, Boulevard du Pont d'Arve 40, CH- 1211 Genève 4, Switzerland

2 School of Psychology, University of Aberdeen, Aberdeen, UK

3 Center for Adaptive Rationality, Max Planck Institute for Human Development, Berlin, Germany

4 Center for the Interdisciplinary Study of Gerontology and Vulnerability (CIGEV), University of Geneva, Geneva, Switzerland

5 Swiss National Center of Competences in Research LIVESOvercoming vulnerability: life course perspectives,

Geneva, Switzerland
Remembering to call a friend for his birthday, taking one's medication at the right moment, or realizing to add an attachment to an e-mail before sending it are typical examples of prospective memory tasks. Prospective memory (PM) refers to remembering to carry out intended actions at an appropriate point in the future while being typically engaged in other ongoing activities (McDaniel \& Einstein, 2007). Prospective remembering can be characterized by different phases (Kliegel, Martin, McDaniel, \& Einstein, 2002; Kliegel, McDaniel, \& Einstein, 2000; Kvavilashvili \& Ellis, 1996). First, the intended action needs to be encoded together with the appropriate moment to execute it. Second, this cue-action plan needs to be maintained during a delay until the relevant moment approaches in which the target cue is detected and the action retrieved. Finally, the intended action needs to be executed according to the initial plan. PM tasks can vary in characteristics of these phases: For instance, the ongoing activity during the retention phase can be more or less demanding, and the relevant moment for retrieval can be time based (e.g., after a specific time has elapsed or at a specific clock time) or event based (when a specific target event happens: see Einstein \& McDaniel, 1990). Variations in these characteristics have been shown to influence the probability that an intended action is successfully remembered and executed (see McDaniel \& Einstein, 2007, for an overview).

One aspect of this process that has not received much attention in PM research so far is the retention phase between encoding of an intention and its retrieval (Kliegel et al. 2011; Meier \& Zimmermann, 2015). That is, which processes are relevant during the retention phase for successful PM? For eventbased PM tasks, mainly two processing scenarios have been discussed in PM research. The first scenario suggests that individuals focus on the ongoing task and that PM cues might trigger the retrieval of an intended action (Einstein et al., 2005; McDaniel \& Einstein, 2000). Here, no controlled attention processes (such as rehearsal of the PM task or strategic monitoring to 
detect the target cue) might be needed. Consequently, ongoing task processing should hardly be influenced by the additional PM task and thus little or possibly no costs on ongoing tasks (i.e., a difference in accuracy or response times between working only on the ongoing task and working on both the ongoing and PM task simultaneously) might be observed. The described processing scenario seems to emerge in PM tasks that are characterized by salient PM target cues (Brandimonte \& Passolunghi, 1994; Harrison, Mullet, Whiffen, Ousterhout, \& Einstein, 2014), high association between a PM target cue and the intended action (McDaniel, Guynn, Einstein, \& Breneiser, 2004; Pereira, Ellis, \& Freeman, 2012), and a high overlap of the information relevant for the ongoing task and for the PM task (i.e., focal tasks; e.g., Einstein et al., 2005; Maylor, 1996; Mullet et al., 2013; Rendell, McDaniel, Forbes, \& Einstein, 2007). Some researchers have argued that in focal tasks (i.e., if an ongoing task involves the processing of the PM target cues), the intended action can be triggered without need for extra attentional monitoring resources (e.g., Scullin, McDaniel, \& Einstein, 2010). An example of a focal task could be that while naming pictures of famous people, the PM task would require a response to pictures of people called John (Rendell et al., 2007). In this scenario, PM performance is typically high, and older and younger adults show similar levels of PM performance (e.g., Kliegel, Jäger, \& Phillips, 2008).

The other scenario is that individuals actively work on both the ongoing task and the PM task simultaneously and therefore strategically deploy considerable attention toward the PM task in order to manage the dual-task constraints (Bisiacchi, Schiff, Ciccola, \& Kliegel, 2009; Bisiacchi, Tarantino, \& Ciccola, 2008). This scenario will be the focus of the present study. This processing scenario can be expected in relatively demanding tasks, with PM performance usually being relatively low and costs to the ongoing task being relatively high (Einstein, Smith, McDaniel, \& Shaw, 1997; Marsh, Hicks, \& Cook, 2005). These effects are especially prominent in older adults, given their limited cognitive resources (Craik, 1986; Light, 1991). The key process that has been examined in these settings so far is cue monitoring, which reflects the strategic allocation of attentional resources that are deployed to detect a target cue (Cona, Bisiacchi, \& Moscovitch, 2013; McDaniel, Einstein, \& Rendell, 2008; Scullin, McDaniel, Shelton, \& Lee, 2010). When there is no or only little overlap between ongoing task and PM targets (i.e., nonfocal tasks; in the aforementioned example of naming pictures of famous people, the task could be to respond to pictures with eyeglasses; Rendell et al., 2007) and if ongoing and PM task are treated equally, attentional resources need to be recruited to monitor for PM target cues (Scullin, McDaniel, Shelton, et al., 2010). Different from focal tasks in which processing of the ongoing task can trigger the intention without much monitoring processes being required, nonfocal tasks require these monitoring processes to detect the target cue. This often results in higher ongoing task costs for nonfocal than for focal tasks, and age effects are more pronounced (Ihle, Hering, Mahy, Bisiacchi, \& Kliegel, 2013; Kliegel et al., 2008).

People can flexibly allocate their cognitive resources between the ongoing task and the PM task: Emphasizing the importance of the PM task typically results in lower ongoing task accuracy, especially in older adults or under demanding conditions (Hering, Phillips, \& Kliegel, 2014; Kliegel, Martin, McDaniel, $\&$ Einstein, 2001, 2004). Moreover, when information about the relevant context to detect the target cue is provided, people show a considerable slowing in the ongoing task in this specific context but not so much in other (irrelevant) contexts (see Cohen, Gordon, Jaudas, Hefer, \& Dreisbach, 2016; Kuhlmann \& Rummel, 2014; Marsh, Cook, \& Hicks, 2006). Hence, attention can be flexibly and actively allocated toward a given PM task.

So far, researchers have mainly studied cue characteristics such as focality or distinctiveness, and any change in ongoing task accuracy or response times was directly linked to monitoring processes to detect the PM target cue, being a cost of the attentional resources available to perform the ongoing task. In the present study, we argue that one additional important factor that should be considered in this dual-task context is the maintenance load of the intentions that have to be remembered and executed. Maintenance load refers to retrospective memory retention of the intention (see also Smith \& Bayen, 2006) and is linked to the retrospective-memory-related characteristics of the PM task such as the number and complexity of cues and their associated actions (i.e., cue-action pairs). This aspect of PM has rarely been investigated. Moreover, as the retention phase of the PM process requires both maintaining the intention and monitoring for the right moment to perform the PM task at the same time, it is very surprising that both aspects have only been studied separately so far (e.g., Cohen, Jaudas, \& Gollwitzer, 2008, investigating effects of intention maintenance, and Scullin, McDaniel, Shelton, et al., 2010, investigating effects of focality on monitoring). Conceptually, it seems plausible that the processes related to maintenance and to monitoring may interact to some extent, as they might compete for similar attentional resources. It is one aim of this study to address their possible interplay.

In the majority of PM studies, the maintenance load was kept minimal (e.g., one cue or category, such as the word $d o g$ or the category "animal words" and one clearly mapped action such as "pressing the space bar"). Nevertheless, a few studies indicated that varying the retrospective load influences PM performance and age-related effects: PM performance was impaired (and age differences were larger) when several instead of one single target cue had to be remembered (e.g., Einstein, Holland, McDaniel, \& Guynn, 1992; but see Otani et al., 1997, for same performance in the study of only younger adults). Moreover, ongoing task costs were larger for three or more PM target cues than for one or two (Cohen et al., 2008). Relatedly, enlarging the delay between encoding and retrieval resulted in lower PM performance (Einstein, McDaniel, Manzi, Cochran, \& Baker, 2000)-particularly in 
older adults (Kelly, Hertzog, Hayes, \& Smith, 2013; McDaniel, Einstein, Stout, \& Morgan, 2003; but not in Einstein et al., 1992). Further, complex PM instructions as well as varying PM target cues many times throughout the experiment impaired PM performance of older adults (Vogels, Dekker, Brouwer, \& de Jong, 2002).

Taken together, PM performance may crucially depend on how much cue monitoring a task requires and how difficult it is to maintain intended action(s). While both aspects have so far been studied separately, hardly anything is known about the relation between monitoring and maintenance demands in event-based PM. Conceptually, examining the relation of these two aspects seems highly important because it illuminates the interplay between memory-related and executive processes, which very likely both underlie ongoing task costs. From a developmental perspective, this interplay appears to be particularly relevant in older adults. In light of cognitive resource limitations (Craik, 1986; Light, 1991), the cognitive aging literature points to age-related impairments in both memory-related and executively demanding tasks.

Theoretically, two outcomes are conceivable: First, possibly, processes related to intention maintenance and to cue monitoring represent independent aspects in PM tasks (and thus may vary independently, affecting PM performance in an additive fashion). In this case, one would assume that higher maintenance load and nonfocal PM tasks might decrease PM performance in a similar way. However, different levels of maintenance load would not influence the focality effect and vice versa. This pattern of outcome seems to be implicitly assumed for the most part of the PM literature: Focality in particular is discussed as the key task feature that determines ongoing task costs (Einstein et al., 2005) and current PM models do not assume any interactions between focality and other task features (e.g., McDaniel \& Einstein, 2000, 2007; McDaniel et al., 2008).

Secondly, on the other hand, current working memory models suggest that maintenance and processing components compete for the same attentional capacity (i.e., resourcesharing conception). As a consequence, one might assume that cue monitoring is moderated by maintenance load (see, e.g., time-based resource-sharing model of working memory; Barrouillet, Bernardin, \& Camos, 2004; Barrouillet, Bernardin, Portrat, Vergauwe, \& Camos, 2007; Vergauwe, Barrouillet, \& Camos, 2010). Two different interactions are conceivable: If high maintenance load conditions would affect preretrieval processes (for example, via periodical intention refreshing in the delay interval), one would expect an effect on preparatory monitoring processes: Fewer attentional resources would be available to monitor for target cues, which should especially affect nonfocal cues relying on these processes in order to be retrieved. Focal cues, however, might still be retrieved with little monitoring (e.g., by a more spontaneous prompting of the PM task). In this case, one would predict an interaction between maintenance load and focality, resulting in larger PM differences between focal and nonfocal tasks in high than low maintenance load conditions (i.e., when maintenance load is high, PM performance would be especially impaired in nonfocal tasks requiring attentional resources for monitoring compared to relatively high PM performance in the focal tasks relying on less monitoring).

The other possibility is that a high maintenance load would particularly affect postretrieval processes (e.g., see Marsh, Hicks, \& Watson, 2002, for details). Here, one would expect that in a first step, focal cues would be detected more easily than nonfocal cues during the ongoing task (i.e., less controlled attention required). In a second step, however, due to high maintenance load, there may not be sufficient attentional capacity to realize the significance of those (better detected) cues and to retrieve and initiate the correct action (McDaniel $\&$ Einstein, 2011). As these postretrieval processes are resource demanding (inhibition of the ongoing task and switch from the ongoing task to the PM task; see Bisiacchi et al., 2009; Bisiacchi et al., 2008; Ihle et al., 2013; Kliegel et al., 2011), there might not be enough attentional resources available for these response management processes. Highmaintenance load might therefore result in low PM performance for both focal and nonfocal cues, independently of their previous (successful) retrieval. Consequently, the interaction would result in a restrained focality effect under high maintenance demands. The present study set out to test these possibilities for the first time. Further, the present study aimed at investigating whether the outlined scenarios would be similar for both younger and older adults or whether they would vary with age, resulting in stronger effects for older adults in the high-maintenance and nonfocal conditions.

\section{Method}

\section{Participants}

Eighty-nine younger (19-32 years) and 90 older adults (60-81 years) participated in the study. The study was approved by the Human Research Ethics Committee of the University of Geneva. Younger adults were students from the University of Geneva. Older adults were recruited via flyers and advertisement in local senior citizen organizations and community centers. Younger adults received course credits; older adults received a monetary reimbursement of CHF 15. Exclusion criteria were neurological and cognitive impairments and the use of psychotropic drugs. Older adults were prescreened for these criteria with the help of the French version of the modified telephone interview for cognitive status (F-TICS-m; Vercambre et al., 2010; Welsh, Breitner, \& Magruderhabib, 1993). Only participants who did not meet the exclusion criteria and who scored at least 28 points in the F-TICS were included in the study. Participants with inaccurate 
retrospective memory for task instructions and inaccurate color perception, as well as participants whose accuracy in the baseline one-back ongoing task block was statistically indistinguishable from chance level ${ }^{1}$ were excluded from the analyses. Seven older and five younger participants did not meet these requirements. The final sample ${ }^{2}$ consisted of 84 younger $\left(M_{\text {age }}=22.24\right.$ years, $S D_{\text {age }}=2.88,69$ female $)$ and 83 older adults $\left(M_{\text {age }}=67.61\right.$ years, $S D_{\text {age }}=5.62,64$ female). Participants' test scores of measures of fluid abilities, vocabulary, and processing speed as well as inferential statistics are displayed in Table 1. As expected, younger adults showed higher scores on fluid intelligence and on processing speed, whereas older adults outperformed younger adults on the vocabulary test.

\section{Design}

We used a $2 \times 2 \times 2$ mixed-factorial design with betweensubject factors age (younger, older adults) and focality ${ }^{3}$ (focal vs. nonfocal task), and within-subjects factor maintenance load (higher vs. lower load). The order of the within-subject condition was counterbalanced; participants were randomly assigned to an order and to an experimental focality condition.

\section{Materials}

Stimuli During the task, participants saw letters (five vowels, A, E, I, O, U, and five consonants, D, H, K, L, M), displayed sequentially. The letters were surrounded by colored frames (i.e., yellow, orange, pink, red, blue, bright green, dark green, purple, gray, brown).

Ongoing task For the ongoing task, participants worked on a category $n$-back task in which they had to decide on each trial whether or not a presented letter belonged to the same letter category (vowel or consonant) as the preceding letter (see Fig. 1). Responses were given by pressing a yes or a no key (on $50 \%$ of all trials, the correct response was yes). Participants worked on five task blocks, each including 60 trials. Each item was presented until a response was given or until $5 \mathrm{~s}$ elapsed (whatever occurred earlier), followed by the next trial. During the first block, participants worked only on the ongoing task as a baseline. For all subsequent blocks, a PM task was added. Two consecutive blocks (second and third as well as fourth and fifth block) belonged to the same experimental condition.

\footnotetext{
${ }^{1}$ We calculated binominal tests for each participant, assuming the responses to the ongoing task were given randomly. This resulted in a performance of $60 \%$, which then was used as a cutoff level.

${ }^{2}$ Note that this final sample was not different from the original one with regard to age, verbal knowledge, fluid intelligence, and processing speed.

${ }^{3}$ Note that we did not aim at examining spontaneous versus strategic PM retrieval but were focusing on the comparison of more or less intense cuemonitoring conditions, within a paradigm that was supposed to require controlled attention.
}

PM task Participants were instructed to complete an additional task that consisted of remembering to press a specified key whenever one of two predefined cues would appear. Participants were instructed to do so before responding to the ongoing task but that the two tasks would be equally important. In the focal group, the predefined target cues were the letters $A$ and $D$, which they were processing for the verbal ongoing task already. In the nonfocal group, participants had to remember to initiate the prospective action when the frame around the letters (which was irrelevant for the ongoing task) turned blue or pink. Keyboards with stickers of all 10 letters and colors presented in the course of the ongoing task were used. These stickers were stuck on a square of 10 keys, and participants were asked to only use those keys for their responses. The positions of the target keys were the same for the letters and colors in order to assure comparability of the focal and nonfocal condition and to avoid advantages for the well-known positions of letters on the keyboard.

In the condition of low maintenance load, participants had to press a key that was marked with the same letter or color of the frame as the target cue (i.e., in focal conditions: press key with $A$ for $A$ as target letter and press key with $D$ for $D$ as target letter; for nonfocal conditions: press key with blue sticker for blue frame and press pink key for pink frame). Thus, the cue-action pairings were relatively simple to remember. For the high-maintenance load condition, participants had to press an unrelated key when detecting the target cue (i.e., in focal conditions: press key with $K$ for $A$ as target letter and press key with $I$ for $D$ as target letter; for nonfocal conditions: press key with gray sticker for blue frame and press green key for pink frame). Thus, there was substantial maintenance load in order to keep the related actions in mind.

Each block of 60 ongoing task trials comprised four PM cues (presented on average every 15 th trial); thus, eight PM cues were presented per maintenance load condition. Only those responses to the target cues that used the correct key and those that were made before responding to the ongoing task were scored as correct PM responses.

Screening To screen for cognitive impairment in the sample of older adults before study inclusion, a French version of the F-TICS-m (Brandt et al., 1993; Welsh et al., 1993) was used. This valid instrument for assessing the overall cognitive status (Vercambre et al., 2010) on the phone is based on the MiniMental State examination (MMSE; Folstein, Folstein, \& Mchugh, 1975).

Vocabulary The Mill Hill Vocabulary Scale (Deltour, 1993) assesses verbal knowledge. It consists of 34 items (one practice item, 33 test items). For each item, one target word and six possible synonyms are provided. The participant has to choose the correct synonym out of the six options. Participants receive one point per correct answer, resulting in a maximum of 33 . 
Table 1 Participant characteristics and cognitive test scores

\begin{tabular}{|c|c|c|c|c|c|c|c|}
\hline & \multicolumn{2}{|c|}{ Younger adults $(N=84)$} & \multicolumn{2}{|c|}{ Older adults $(N=83)$} & \multirow[b]{2}{*}{$t$} & \multirow[b]{2}{*}{$d f$} & \multirow[b]{2}{*}{ Cohen's $d$} \\
\hline & $M$ & $S D$ & $M$ & $S D$ & & & \\
\hline Fluid abilities $^{\mathrm{a}}$ & 7.55 & 2.04 & 4.83 & 2.28 & 8.11 & 165 & 1.26 \\
\hline Vocabulary $^{\mathrm{b}}$ & 22.89 & 3.42 & 26.60 & 4.71 & -5.82 & 149.50 & 0.90 \\
\hline Processing speed ${ }^{\mathrm{c}}$ & 64.06 & 9.55 & 45.60 & 9.06 & 12.77 & 165 & 1.98 \\
\hline
\end{tabular}

${ }^{\text {a }}$ Short version of Raven's Matrices Test

${ }^{\mathrm{b}}$ Mill Hill Vocabulary Test

${ }^{\mathrm{c}}$ Digit-Symbol Substitution Test

Fluid intelligence This short version of the Advanced Progressive Raven Matrices Test to assess higher order general cognitive abilities was validated by Arthur and Day (1994) and consists of 12 items with advancing difficulty. A $3 \times 3$ array is presented with eight icons and one empty field. Participants have to choose one out of eight figures to fill in the empty space that best continues the line of icons according to an underlying logical rule.

Processing speed To assess processing speed, the Coding subtest of the Wechsler Adult Intelligence Scale (WAIS-IV; Wechsler, 2011) was administered. In this digit symbol substitution test, subjects are presented with symbols that are matched to the numbers from one to nine as well as a testing sheet that displays 133 numbers in random order. The task consists of copying the respective symbol under each digit in a sequential order. Subjects are asked to work as fast and accurate as possible. The correct symbols recorded within the time limit (90 seconds) are added up as performance measure (133 points maximum).

\section{Procedure}

After providing informed consent, participants received instructions for the ongoing task, followed by two training runs: one with feedback and a bit slower than the regular trials (10 trials) and the second run being as fast as the regular trials and without feedback (20 trials). Only participants that reached at least $60 \%$ correct answers could continue, otherwise the training session was repeated until they would reach this threshold.

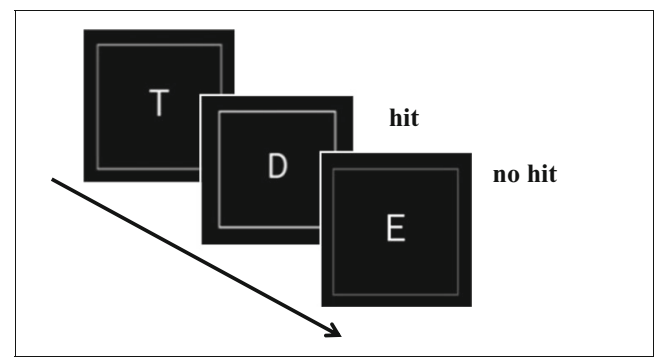

Fig. 1 Ongoing task used in the present study: One-back letter category task
A test block of the ongoing task followed. PM instructions for one of the two PM conditions were provided as the next step, ensued by a delay of 5 minutes that was filled with performing the other background tests (see below). Then, one PM block started. This procedure was the same for the next three blocks: PM and ongoing task instructions were repeated, then a delay of 5 minutes followed before the next PM block started without repeating the PM instruction again. Within these breaks, the background questionnaire for the younger adults (older adults completed it on the phone in advance), the vocabulary test, the Raven test and the Coding test were performed sequentially. If participants did not complete a test within these 5 minutes, it was continued in the subsequent delay. After completing all test blocks, a questionnaire asking for task difficulty and retrospective memory for the PM task was administered, and participants in the nonfocal condition additionally completed a posttest to verify the correct perception of the cue colors. Participants got either course credit or were reimbursed.

\section{Results}

The alpha level was set to .05. Significant interactions were explored using post hoc $t$ tests and pairwise comparisons between specific conditions of the experimental factors. Post hoc tests were corrected for multiple testing using the Bonferroni correction by multiplying the $p$ values of each post hoc test by the number of conditions (Maxwell \& Delaney, 2004).

\section{PM performance}

To analyze PM performance, the proportion of correct PM responses was calculated for each experimental condition (see Fig. 2). A 2 (age: young vs. old) $\times 2$ (focality: focal vs. nonfocal) $\times 2$ (maintenance load: high vs. low) mixed-factorial ANOVA was used to compare PM performance. Analyses revealed a significant effect of maintenance load, $F(1,163)=25.84, p<.001$, $\eta_{\mathrm{p}}{ }^{2}=.14$, suggesting higher PM performance for lowmaintenance load $(M=.71, S D=.28)$ than high-maintenance 


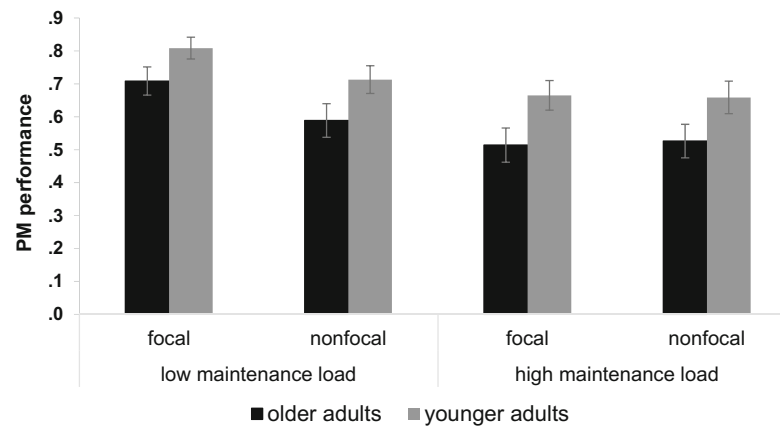

Fig. 2 Average PM performance (proportions of hits). Error bars represent $+/-1 S E$

load $(M=.59, S D=.32)$. The effect of focality failed to reach significance, $F(1,163)=1.68, p=.20, \eta_{\mathrm{p}}{ }^{2}=.01$, but was present only in the low-maintenance load condition, indexed by a significant interaction of maintenance load and focality, $F(1,163)=$ $6.13, p=.01, \eta_{\mathrm{p}}^{2}=.04$. Post hoc tests showed that for lowmaintenance load, performance on focal tasks was higher than on nonfocal tasks, $t(165)=2.55, p_{\text {adj }}=.01$. This difference was not present in the high-maintenance load condition, $t<1$.

There was a significant main effect of age, $F(1,163)=9.90$, $p=.002, \eta_{\mathrm{p}}^{2}=.06$, with higher PM performance for younger adults $(M=.71, S D=.24)$ than for older $(M=.59, S D=.28)$. Interactions of age with any of the two other factors and the triple interaction of all factors were not significant (all $F_{\mathrm{s}}<1$ ).

To additionally investigate PM confusion errors (correct detection of PM target cue but press of wrong target key), we analyzed the proportion of false PM responses to the PM target cues. As before, a 2 (age: young vs. old) $\times 2$ (focality: focal vs. nonfocal) $\times 2$ (maintenance load: high vs. low) mixed-factorial ANOVA was applied to compare confusion errors. Analyses revealed a main effect of maintenance load, $F(1,163)=15.77, p<.001, \eta_{\mathrm{p}}^{2}=.09$, indicating a higher proportion of confusion errors in the high- $(M=.048, S D=$ $.132)$ than in the low-maintenance load condition $(M=.005$, $S D=.025)$. All other main effects and interactions did not reach significance $(p s>.066)$.

\section{Ongoing task accuracy}

The proportion of correct responses to the ongoing task was calculated separately for each of the five blocks (i.e., one ongoing task only and four PM blocks). The first two trials of each block and the two trials following a PM trial were not taken into account. A 2 (age: young vs. old) $\times 2$ (focality: focal vs. nonfocal) $\times$ 3 (experimental block: ongoing only, high load, low load) mixedfactorial ANOVA was conducted to compare ongoing task accuracy (see Table 2). Results revealed a main effect of focality, $F(1$, $163)=4.21, p=.04, \eta_{\mathrm{p}}{ }^{2}=.03$, and a main effect of experimental block, $F(2,326)=32.48, p<.001, \eta_{\mathrm{p}}{ }^{2}=.17$. Post hoc pairwise comparisons showed that ongoing task accuracy differed between the ongoing task only block and the high load condition $(p<.001)$ as well as the high and low load conditions $(p<.001)$, but not between the ongoing only block and the low load condition $(p>.99)$. All other effects were not significant $(p s>.11)$.

\section{Ongoing task response time costs}

The costs of adding a PM task to an ongoing task were calculated by subtracting each participant's mean response time in the ongoing-task-only block from the mean response time of the ongoing task trials in each PM block. Following previous research, response times of incorrect ongoing task trials, of PM trials, and of the two trials following each target cue, were omitted from analyses (for similar procedure, see Boywitt \& Rummel, 2012; Brewer, 2011; Cohen et al., 2016; Horn, Bayen, \& Smith, 2013). A mixed-factorial ANOVA indicated significant main effects of maintenance load, $F(1,163)=$ $26.95, p<.001, \eta_{\mathrm{p}}^{2}=.14$, focality, $F(1,163)=5.82, p=.02$, $\eta_{\mathrm{p}}{ }^{2}=.03$, and age, $F(1,163)=10.24, p<.01, \eta_{\mathrm{p}}{ }^{2}=.06$, reflecting higher ongoing task response time costs in the highcompared to the low-maintenance load condition, nonfocal tasks compared to focal tasks and higher costs for older adults as compared with younger adults. Further, there was a significant interaction of maintenance load and focality, $F(1,163)=8.55, p$ $<.01, \eta_{\mathrm{p}}^{2}=.05$, as displayed in Fig. 3. Specifically, post hoc tests indicated that while in the low maintenance load condition the usual focality cost effect was present, $t(165)=4.24, p_{\text {adj }}<.001$, this was not the case for the high-maintenance load condition $(t<$ 1). Further, while in the focal condition response time costs were different between the two maintenance load conditions, $t(91)=$ $6.66, p_{\text {adj }}<.001$, this was not true for the nonfocal task $t(74)=$ $1.39, p_{\text {adj }}=.34$. The interactions of age and maintenance load, age and focality, as well as the triple interaction of all factors did not reach significance $(p \mathrm{~s}>.14){ }^{4}$

\footnotetext{
${ }^{4}$ To explore possible order effects and their interaction with the factors maintenance load, focality, and age, we performed additional ANOVAs on ongoing task accuracy and response times including order of the maintenance load conditions as additional factor. Analyses of ongoing task accuracy revealed an interaction of order and experimental block (i.e., ongoing task only, high load, low load) with especially lower accuracy when the high-maintenance load condition was performed prior to the low-maintenance load condition. Similarly, analyses of ongoing task response times revealed a main effect of order, with greater costs in the first compared to the second block, as well as an interaction of order with maintenance load (greater costs when the highmaintenance load condition was performed before the low-maintenance condition), and a triple interaction of order, maintenance load, and focality. To test the robustness of the initial aforementioned analyses and to investigate costs while accounting for carryover or practice effects, we analyzed the data from the first PM block that each participant performed (high- or low-maintenance load condition) and compared performance in these blocks using betweensubject ANOVAs. Analyses of ongoing task accuracy showed the same pattern as when compared within-subject (see above). Additionally, experimental block and focality interacted, indicating a greater reduction of ongoing task accuracy for nonfocal than for focal tasks. Results of the ongoing task response times showed exactly the same pattern as when conducted within-subject with similar or slightly higher effect sizes.
} 
Table 2 Means and standard deviations of ongoing task accuracy (proportions of correct responses in the one-back task) for younger and older adults

\begin{tabular}{lllllll}
\hline & \multicolumn{3}{l}{ Younger adults } & & & \multirow{2}{*}{ Older adults } \\
\cline { 2 - 3 } Experimental Block & Focal $M(S D)$ & & Nonfocal $M(S D)$ & & Focal $M(S D)$ & Nonfocal $M(S D)$ \\
\hline Ongoing task only & $.928(.049)$ & $.907(.069)$ & & $.913(.069)$ & $.921(.052)$ \\
Ongoing task + low-maintenance load PM task & $.933(.054)$ & $.906(.051)$ & & $.931(.073)$ & $.899(.084)$ \\
Ongoing task + high-maintenance load PM task & $.899(.078)$ & $.878(.091)$ & & $.876(.091)$ & $.858(.088)$ \\
\hline
\end{tabular}

Additionally, to investigate the occurrence of absolute PM costs in the low-maintenance, focal condition, costs of both younger and older adults were analyzed using separate onesample $t$ tests. For both older, $t(44)=5.05, p<.01$, and younger adults, $t(46)=4.04, p<.01$, analyses revealed substantial costs that were different from zero.

\section{Exploratory analyses}

To control for the impact of working memory on the observed effects on PM performance, an ANCOVA was conducted using performance of the block where only the $n$-back ongoing task was performed as a covariate. There was a significant effect of one-back performance, with the result that in comparison to the aforementioned results, the effect of maintenance load on PM performance was no longer significant, $F(1,162)=0.13, p=.71, \eta_{\mathrm{p}}{ }^{2}<.01$ None of the other observed effects on PM performance changed when controlling for working memory.

\section{Discussion}

The present study set out to investigate the impact of maintenance load, focality, and age on PM performance, ongoing task accuracy as well as on ongoing task response time costs. Furthermore, for the first time, we aimed at disentangling the possible interplay of maintenance load and focality of the PM task.

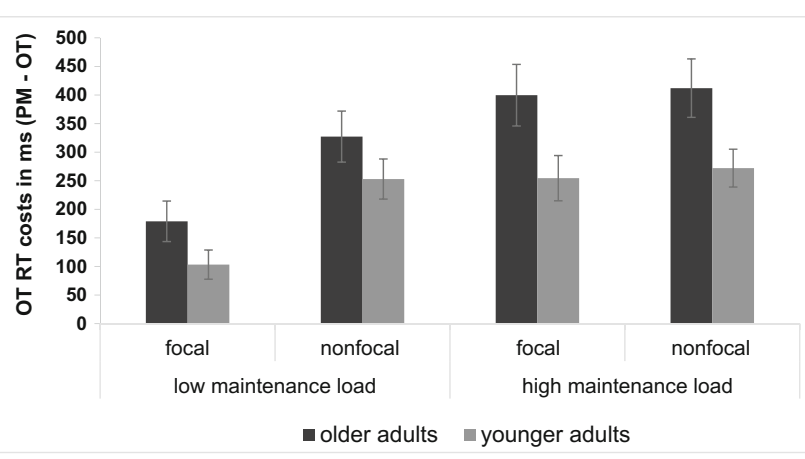

Fig. 3 Ongoing task (OT) response time costs (RTs PM blocks-RTs ongoing task only block). Error bars represent + - $1 S E$
Results suggest a high impact of maintenance load and age on PM performance, ongoing task accuracy (maintenance load only), and ongoing task costs. Notably, in the present paradigm, focality did not generally affect PM performance but affected ongoing task accuracy and response time costs. More precisely, focality only had an impact on PM performance when maintenance load was low (but not when it was high). The same interaction pattern was present for ongoing task costs (advantage of focal compared to nonfocal tasks only in low-maintenance load condition, high costs for both focal and nonfocal tasks in the high-maintenance load condition). Age did not interact with any of the other factors, neither regarding PM or ongoing task accuracy nor ongoing task response time costs. Confusion errors were only affected by maintenance load, with them being higher in the highmaintenance than in the low-maintenance load condition. Besides, exploratory analyses showed that if working memory performance was taken into account, the strong effect of maintenance load on PM performance was not present anymore.

First of all, the strong effect of maintenance load of the intended action on PM performance and on PM confusion errors confirmed a conceptually important impact of memory-related factors on PM. This finding is in line with some studies that showed reduced PM performance when the complexity of the PM task was manipulated by increasing the number of target cues (e.g., Einstein et al., 1992), by enlarging the length of the delay between encoding and retrieval (Einstein et al., 2000), or by introducing a PM instruction that caused high load (Vogels et al., 2002). So far, however, most laboratory research only used very simple action(s) to be kept in mind, resulting in low maintenance load. Additionally, many studies excluded participants who did not recall the intended action correctly, which has possibly masked effects of maintenance load on PM performance. The present research clearly highlights the so far possibly underestimated importance to further investigate the impact of memory-related factors on PM performance.

How can this effect be explained? Exploratory analyses revealed that when considering individual working memory capacity, the strong effect of maintenance load on PM performance disappeared. This finding gives some indication of possible process mechanisms of maintenance on PM. It is likely that in order to sustain the high maintenance load, working memory resources are drained, for instance, to periodically 
rehearse or refresh the actions related to the target cues. Depending on an individual's working memory capacity, these resources might have been more or less depleted, which could have resulted in subjects being more or less affected by high-maintenance load. This is in line with Kliegel and Jäger (2006), who also showed working memory resources being related to maintaining an intention over a delay interval. In that way, also other maintenance load manipulations should result in similar findings, as long as they require the periodic recruitment of working memory capacities to refresh the PM task representation and if the high-maintenance load condition exceeds a certain amount of information to be kept in mind. Accordingly, Cohen et al. (2008) showed impaired ongoing costs when three but not one or two PM cues had to be remembered. In general, different manipulations might require differently strong variations to affect maintenance load (by exceeding working memory capacities), but we expect any variation of maintenance characteristics of cues and/or actions to show similar results. Possibilities are varying the number of PM cues, the number of different actions, the mapping between cues and actions, the complexity of cues or actions (e.g., single color vs. complex configuration of color), shape, background of the target cue, or any additional specificities to remember the cue or/and action (e.g., changing keys to respond to target cue: first time press $A$, second time $E$, then $O$, and so on). However, at this stage, the assumption of similar effects of these factors on PM remain speculative, and future studies that investigate different maintenance load manipulations and their impact on PM and underlying mechanisms are required.

Focality did not impact PM performance but affected ongoing task accuracy and response time costs. We acknowledge that our focality manipulation somewhat deviates from traditional manipulations in placing the nonfocal cue also in the periphery of the visual focus. Nevertheless, a trade-off between PM performance and ongoing task costs has already been shown by other studies, such as Cona et al. (2013), reflecting a stronger investment of attentional resources in the nonfocal condition in order to maintain high performance. In line with this, our findings suggest that focality primarily impacts the allocation of strategic resources to the task (top-down processes), namely, a higher investment of resources to monitor the environment for the target cue in nonfocal (compared to focal) tasks (Einstein et al., 2005; Scullin, McDaniel, Shelton, et al., 2010). This flexible investment of attentional resources in response to particular task characteristics (such as focality affecting ongoing task measures in the present study) has similarly been shown when importance of the PM task was enhanced (Hering et al., 2014; Kliegel et al., 2001; Kliegel, Martin, et al., 2004) or when information about the relevant context for the PM task was provided (Cohen et al., 2016; Kuhlmann \& Rummel, 2014; Marsh et al., 2006).
Most notably, focality and maintenance load interacted, confirming the second suggested scenario: When the maintenance load was low, the characteristic focality effects reported in former PM literature emerged (i.e., lower PM performance and higher ongoing task costs in nonfocal as compared to focal cues; see, e.g., Einstein et al., 2005; Rendell et al., 2007; Scullin, McDaniel, Shelton, et al., 2010). By contrast, if maintenance load was high, there was no advantage of focal over nonfocal tasks, neither with regard to PM performance nor to ongoing task costs. Conceptually, this interaction is in line with current working memory models (Barrouillet et al., 2004; Barrouillet et al., 2007) that underpin the resourcesharing conception by suggesting that maintenance and processing components compete for the same attentional resources. It seems that when resources have been drained for maintenance processes, they are not available anymore to profit from less demanding retrieval conditions in focal tasks. This interaction pattern suggests that postretrieval rather than preretrieval processes are affected: Even though in focal tasks the required target information is processed (so that the target cues should be detected more easily than in nonfocal tasks), this advantage in cue detection does not emerge when maintenance load is high. What could be the reason? It is likely that, as the maintenance load differences were related to working memory capacity, a higher maintenance load might have caused a working memory overload. For that reason, fewer cognitive resources might have been at disposal to note the significance of the detected cues or to inhibit the ongoing task in order to execute the intended action. These postretrieval processes have been shown to be executively demanding (Bisiacchi et al., 2009; Bisiacchi et al., 2008; Ihle et al., 2013), especially coordinating the subprocesses seem to produce additive costs (Marsh et al., 2002). More precisely, these processes require task switching and inhibitory capacities, which, due to the high retrospective-memory load, might not be sufficiently available. Crucially, this result suggests that the focality effect that has been established in PM literature might only emerge if the maintenance load would be (sufficiently) low and hence if sufficient working memory resources would be available for the postretrieval processes (at least for the focality manipulation used in the present study).

The interaction of focality with maintenance load is of theoretical importance, as to date interactions between different factors influencing PM have not been described in a conceptual way. This was neither the case in the original version of the multiprocess theory (McDaniel \& Einstein, 2000) nor in its revised version (Scullin et al. 2013), and thus the interaction finding has the potential to extend fundamental theories in PM. Furthermore, the two process model of strategic monitoring (Guynn, 2003) suggests that monitoring entails the subprocesses retrieval mode and target checking. Although we did not aim to directly test these processes, and the link of our results to that view remains speculative, our results may 
indicate that a retrieval mode (i.e., a readiness to perform a PM task, accompanied by higher activation of the PM task) was especially strong for the high-maintenance load conditions, as keeping the complex cue-action pair in mind might enhance the activation of the PM task (e.g., by refreshing the cueaction combination). Target checking, by contrast, might be more affected by focality (e.g., with nonfocal tasks amplifying the need to carefully check for target cues). These two processes might affect response time costs in different ways but be drained from the same amount of working memory resources, as reflected by the interaction pattern in the ongoing task costs. More research on the interplay between retrospective memory load and focality as well as other traditional factors impacting PM is required to further investigate that topic. In particular, focality manipulations that were used in prior studies should be applied to relate findings to previously obtained focality effects.

Furthermore, another process that takes place during retention phase in a PM task is the processing of the ongoing task. As the ongoing task can be differently absorbing (resulting in diverse recruitments of resources), it would be interesting to investigate how variations of ongoing task absorption would relate to both maintenance and cue detection processes, as well as to their interplay.

Besides, the present finding is likely to reveal an additional dimension of ongoing task response time costs: While the target cues were the same between the high- and low-maintenance condition within each focality condition (i.e., the focality of the PM task did not change between those two), response time costs differed substantially between the two maintenance conditions. This pattern also held true when controlling for emerging carryover effects. This finding suggests that ongoing task response time costs may not only be related to target monitoring but may reflect maintenance demands, too (Cohen et al., 2008). This additional source of costs should especially be considered in paradigms using complex PM tasks (see also Marsh et al., 2002). Future research is required to disentangle different components of ongoing task response time costs. Besides, based on this finding, it cannot be fully excluded that our maintenance load manipulation did not also affect executive processes, like in the high load condition to inhibit to press the key with the same letter as the target cue and instead press the key with the required target letter.

With regard to age-related differences, younger adults outperformed older adults and generally showed lower ongoing task costs. This replicates many other studies showing age-related impairment in PM (e.g., Park, Hertzog, Kidder, Morrell, \& Mayhorn, 1997; West \& Craik, 2001; for metaanalyses, see Henry, MacLeod, Phillips, \& Crawford, 2004; Ihle et al., 2013). One surprising finding was that age did not interact with any of the other factors, either with maintenance load or with focality. It seems that older adults showed the same pattern of results as the younger ones, with the only difference being that older adults demonstrated an overall lower level of performance and higher costs in general. We expected older adults to be more disadvantaged than younger ones specifically in conditions of high demand and of high monitoring requirement, which was not the case. For instance, it has repeatedly been proposed that focality might underlie age effects in PM performance (Einstein \& McDaniel, 2005; McDaniel et al., 2008), which was not supported by the present data (note, however, that Smith \& Bayen, 2006, as well as Smith \& Hunt, 2014, would suggest preparatory attention throughout conditions, therefore resulting in age effects in all PM tasks, which seems to be supported by the present data and the observation of nonzero costs in both focal and nonfocal tasks). However, as it was the case in the present study, Kelly et al. (2013); Rose, Rendell, McDaniel, Aberle, and Kliegel (2010); and Ihle, Ghisletta, and Kliegel (2017) also did not find an interaction of age and focality. In fact, older adults already showed lower PM performance than the younger adults in low demanding conditions (i.e., in the focal task with low-maintenance load). Similarly, although often with smaller effect sizes than in nonfocal tasks, several other studies also showed age differences in focal tasks (Neulinger, Oram, Tinson, O'Gorman, \& Shum, 2016; Niedzwienska \& Barzykowski, 2012; Reese-Melancon, 2013; Rendell et al., 2007; see also meta-analyses Kliegel et al., 2008; Ihle et al., 2013). Even though age differences were present in the nonfocal tasks, the appearance of clear age differences in focal tasks prevented the finding of a significant interaction of age and focality, since nonfocal tasks did not affect older adults to a greater extent than did focal tasks. Hence, the overall reduced level of resources rather than the interaction of age with more demanding conditions might be accountable for the general age effect. The finding of a missing Age $\times$ Focality interaction can be explained by the specific experimental paradigm used in the present study: The ongoing task was a rather complex one-back working memory task that required attentional resources to be (successfully) processed. In the focal task, subjects additionally had to respond to two specific letters, which in combination with the resources being drained for the ongoing task could have enhanced task difficulty compared prior studies using only one specific PM target cue (e.g., Scullin, McDaniel, \& Einstein, 2010) embedded in a simpler ongoing task. Indeed, substantial costs were found for both younger and older adults in the focal lowmaintenance condition. ${ }^{5}$ For older adults, the rather demanding focal task is likely to have exceeded their available resources, resulting in PM age differences. This finding prompts the conclusion that younger and older adults perform equally in PM tasks only if they exclusively rely on spontaneous

\footnotetext{
$\overline{5}$ However, note that we did not aim at investigating pure spontaneous retrieval, and see that Zuber, Kliegel, and Ihle (2016) could show that to some extent even focal tasks relate to controlled attention.
} 
retrieval (see also Mullet et al., 2013). The nonfocal condition, against this, required to remember two specific colors that surrounded the letters of the ongoing task. This manipulation may have produced a less extreme contrast to the focal task than was the case in other studies with deriving focality manipulations (also using more demanding nonfocal cues like word syllables or target categories; see Einstein \& McDaniel, 2005). The smaller contrast might explain why age effects in the present study were not greater for nonfocal than for focal tasks, thus not resulting in an interaction of age and focality. The present results indicate that a distinction between focal and nonfocal might not always be sufficient to predict performance outcomes, because other task characteristics may also influence PM and age effects. As suggested by Zuber and colleagues (2016), we may have to shape extant theories of the processes contributing to focal versus nonfocal PM. This requires future studies that use different focality and ongoing task manipulations to examine in how far effects are task specific or generalize across different paradigms.

Alternatively, it might be the case that (at least in our sample) the investigated processes are not affected by age. In line with this, Maylor, Smith, Della Sala, and Logie (2002) did not find an interaction of age and cue-action association on PM performance, either, and Cohen, Dixon, Lindsay, and Masson (2003) in their second experiment also did not find differential effects of distinctiveness for young and older adults. Additionally, older adults showed substantially higher ongoing task response time costs than the younger ones even in the focal task with low maintenance load. The higher costs, however, did not result in similar PM performance as in younger adults (as one could expect if they were investing their resources to overcome age-related deficits), indicating a less efficient usage of attentional resources. Nevertheless, it is worth highlighting that both age groups showed the same ongoing task accuracy in all conditions. Possibly, older adults' higher response time costs were more related to maintaining high performance in the demanding ongoing task than in the PM task (therefore perhaps resulting in age effects already in the low demanding condition). From a conceptual point of view, the missing Age $\times$ Focality interaction deserves further exploration.

The lack of an interaction of age and maintenance load contrasts the few prior studies finding age to interact with maintenance load (e.g., Einstein et al., 2000; Kidder, Park, Hertzog, \& Morrell, 1997) but corresponds with findings by Einstein et al. (1992), showing that the length of the delay between encoding and retrieval was not affected by age. Nevertheless, our manipulation differed from studies manipulating the maintenance load by multiple instead of one single-target cues (Einstein et al., 1992; Kidder et al., 1997), a prolonged encoding-retrieval delay (Kelly et al., 2013; McDaniel et al., 2003) or complex PM instructions and varying PM target cues (Vogels et al., 2002) and thus requires further replication. However, it is important to underline that PM confusion errors in our sample were not affected by age, suggesting that processes related to the retrospective component of PM were comparable between younger and older adults and thus not provoking differential age effects in the two maintenance conditions. Conceptually, this lack of differential age effects suggests that the well-documented retrospective memory decline in older adults (Brickman \& Stern, 2009; Verhaeghen \& Salthouse, 1997) cannot fully explain agerelated differences in PM performance. This corresponds with literature showing that intention retention processes (Kliegel, Eschen, \& Thone-Otto, 2004; Kliegel, McDaniel, \& Einstein, $2000)$ as well as the retrospective component of PM (Cohen et al., 2003; but see Zöllig et al., 2007, for the opposite finding) are often unaffected by age.

In conclusion, the present study revealed that PM performance and ongoing task costs were strongly affected by maintenance load and age. Conceptually important, the characteristic focality effects only emerged when maintenance load was low, but not when attentional resources were deployed for maintaining a more demanding intention. These results suggest that maintenance and cue monitoring compete for the same attentional capacity. Hence, the current findings indicate for a first time that high-maintenance load affects postretrieval processes via its impact on working memory resources, which can restrain the advantage of focal over nonfocal tasks.

Acknowledgments We thank Jessica Wildberger, Justine Gouiller, and Anny Molnar Mafra for assistance with data collection and Sascha Zuber and Alexandra Hering for helpful comments on a prior version of the manuscript.

M.K. and K.M.S. acknowledge support from Swiss National Science Foundation.

\section{References}

Arthur, W., \& Day, D. V. (1994). Development of a short-form for the Raven Advanced Progressive Matrices Test. Educational and Psychological Measurement, 54(2), 394-403. doi:10.1177/ 0013164494054002013

Barrouillet, P., Bernardin, S., \& Camos, V. (2004). Time constraints and resource sharing in adults' working memory spans. Journal of Experimental Psychology-General, 133(1), 83-100. doi:10.1037/ 0096-3445.133.1.83

Barrouillet, P., Bernardin, S., Portrat, S., Vergauwe, E., \& Camos, V. (2007). Time and cognitive load in working memory. Journal of Experimental Psychology: Learning, Memory, and Cognition, 33(3), 570-585. doi:10.1037/0278-7393.33.3.570

Bisiacchi, P. S., Schiff, S., Ciccola, A., \& Kliegel, M. (2009). The role of dual-task and task-switch in prospective memory: Behavioural data and neural correlates. Neuropsychologia, 47(5), 1362-1373. doi:10. 1016/j.neuropsychologia.2009.01.034

Bisiacchi, P. S., Tarantino, V., \& Ciccola, A. (2008). Aging and prospective memory: The role of working memory and monitoring processes. Aging Clinical and Experimental Research, 20(6), 569-577. 
Boywitt, C. D., \& Rummel, J. (2012). A diffusion model analysis of task interference effects in prospective memory. Memory \& Cognition, 40(1), 70-82. doi:10.3758/s13421-011-0128-6

Brandimonte, M. A., \& Passolunghi, M. C. (1994). The effect of cuefamiliarity, cue-distinctiveness, and retention interval on prospective remembering. The Quarterly Journal of Experimental Psychology, $47(3), 565-587$.

Brandt, J., Welsh, K. A., Breitner, J. C. S., Folstein, M. F., Helms, M., \& Christian, J. C. (1993). Hereditary influences on cognitivefunctioning in older men-A study of 4000 twin wairs. Archives of Neurology, 50(6), 599-603.

Brewer, G. A. (2011). Analyzing response time distributions methodological and theoretical suggestions for prospective memory researchers. Zeitschrift Für Psychologie-Journal of Psychology, 219(2), 117-124. doi:10.1027/2151-2604/a000056

Brickman, A. M., \& Stern, Y. (2009). Aging and memory in humans. Encyclopedia of Neuroscience, 1, 175-180.

Cohen, A.-L., Dixon, R. A., Lindsay, D. S., \& Masson, M. E. (2003). The effect of perceptual distinctiveness on the prospective and retrospective components of prospective memory in young and old adults. Canadian Journal of Experimental Psychology-Revue Canadienne De Psychologie Experimentale, 57(4), 274-289.

Cohen, A.-L., Gordon, A., Jaudas, A., Hefer, C., \& Dreisbach, G. (2016). Let it go: The flexible engagement and disengagement of monitoring processes in a non-focal prospective memory task. Psychological Research. doi:10.1007/s00426-016-0744-7

Cohen, A.-L., Jaudas, A., \& Gollwitzer, P. M. (2008). Number of cues influences the cost of remembering to remember. Memory \& Cognition, 36(1), 149-156.

Cona, G., Bisiacchi, P. S., \& Moscovitch, M. (2013). The effects of focal and nonfocal cues on the neural correlates of prospective memory: Insights from ERPs. Cerebral Cortex. doi:10.1093/cercor/bht116

Craik, F. I. M. (1986). A functional account of age differences in memory. In F. Klix \& H. Hagendorf (Eds.), Human memory and cognitive capabilities (pp. 409-422). Amsterdam, Netherlands: Elsevier.

Deltour, J. J. (1993). Echelle de vocabulaire Mill Hill de J. C. Raven: Adaptation française et normes comparées du Mill Hill et du Standard Progressive Matrices (PM38): Manuel [Mill Hill Vocabulary Scale of J. C. Raven: French Adaptation and comparative norms of Mill Hill and the Standard Progressive Matrices (PM38). Manual]. Braine-le-Château, Belgium: L'application des techniques modernes.

Einstein, G. O., Holland, L. J., McDaniel, M. A., \& Guynn, M. J. (1992). Age-related deficits in prospective memory: The influence of task complexity. Psychology and Aging, 7(3), 471-478.

Einstein, G. O., \& McDaniel, M. A. (1990). Normal aging and prospective memory. Journal of Experimental Psychology: Learning, Memory, and Cognition, 16(4), 717-726.

Einstein, G. O., \& McDaniel, M. A. (2005). Prospective memory: Multiple retrieval processes. Current Directions in Psychological Science, 14(6), 286-290. doi:10.1111/j.0963-7214.2005.00382.x

Einstein, G. O., McDaniel, M. A., Manzi, M., Cochran, B., \& Baker, M. (2000). Prospective memory and aging: forgetting intentions over short delays. Psychology and Aging, 15(4), 671-683.

Einstein, G. O., McDaniel, M. A., Thomas, R., Mayfield, S., Shank, H., Morrisette, N., \& Breneiser, J. (2005). Multiple processes in prospective memory retrieval: Factors determining monitoring versus spontaneous retrieval. Journal of Experimental PsychologyGeneral, 134(3), 327-342. doi:10.1037/0096-3445.134.3.327

Einstein, G. O., Smith, R. E., McDaniel, M. A., \& Shaw, P. (1997). Aging and prospective memory: The influence of increased task demands at encoding and retrieval. Psychology and Aging, 12(3), 479-488. doi:10.1037/0882-7974.12.3.479

Folstein, M. F., Folstein, S. E., \& Mchugh, P. R. (1975). "Mini-Mental State": A practical method for grading the cognitive state of patients for the clinician. Journal of Psychiatric Research, 12(3), 189-198. doi:10.1016/0022-3956(75)90026-6

Guynn, M. J. (2003). A two-process model of strategic monitoring in event-based prospective memory: Activation/retrieval mode and checking. International Journal of Psychology, 38(4), 245-256. doi:10.1080/00207590244000205

Harrison, T. L., Mullet, H. G., Whiffen, K. N., Ousterhout, H., \& Einstein, G. O. (2014). Prospective memory: Effects of divided attention on spontaneous retrieval. Memory \& Cognition, 42(2), 212-224. doi: 10.3758/s13421-013-0357-y

Henry, J. D., MacLeod, M. S., Phillips, L. H., \& Crawford, J. R. (2004). A meta-analytic review of prospective memory and aging. Psychology and Aging, 19(1), 27-39. doi:10.1037/0882-7974.19.1.27

Hering, A., Phillips, L. H., \& Kliegel, M. (2014). Importance effects on age differences in performance in event-based prospective memory. Gerontology, 60(1), 73-78. doi:10.1159/000355057

Horn, S. S., Bayen, U. J., \& Smith, R. E. (2013). Adult age differences in interference from a prospective-memory task: A diffusion model analysis. Psychonomic Bulletin \& Review, 20(6), 1266-1273. doi: 10.3758/s13423-013-0451-y

Ihle, A., Ghisletta, P., \& Kliegel, M. (2017). Prospective memory and intraindividual variability in ongoing task response times in an adult lifespan sample: The role of cue focality. Memory, 25(3), 370-376. doi:10.1080/09658211.2016.1173705

Ihle, A., Hering, A., Mahy, C. E., Bisiacchi, P. S., \& Kliegel, M. (2013). Adult age differences, response management, and cue focality in event-based prospective memory: A meta-analysis on the role of task order specificity. Psychology and Aging, 28(3), 714-720. doi: $10.1037 / \mathrm{a} 0033653$

Kelly, A. J., Hertzog, C., Hayes, M. G., \& Smith, A. D. (2013). The effects of age and focality on delay-execute prospective memory. Aging, Neuropsychology, and Cognition, 20(1), 101-124. doi:10. 1080/13825585.2012.691152

Kidder, D. P., Park, D. C., Hertzog, C., \& Morrell, R. W. (1997). Prospective memory and aging: The effects of working memory and prospective memory task load. Aging Neuropsychology and Cognition, 4(2), 93-112. doi:10.1080/13825589708256639

Kliegel, M., Altgassen, M., Hering, A., \& Rose, N. S. (2011). A processmodel based approach to prospective memory impairment in Parkinson's disease. Neuropsychologia, 49(8), 2166-2177. doi:10. 1016/j.neuropsychologia.2011.01.024

Kliegel, M., Eschen, A., \& Thone-Otto, A. I. (2004). Planning and realization of complex intentions in traumatic brain injury and normal aging. Brain and Cognition, 56(1), 43-54. doi:10.1016/j.bandc.2004.05.005

Kliegel, M., \& Jäger, T. (2006). Delayed-execute prospective memory performance: The effects of age and working memory. Developmental Neuropsychology, 30(3), 819-843. doi:10.1207/ s15326942dn3003 4

Kliegel, M., Jäger, T., \& Phillips, L. H. (2008). Adult age differences in event-based prospective memory: A meta-analysis on the role of focal versus nonfocal cues. Psychology and Aging, 23(1), 203208. doi:10.1037/0882-7974.23.1.203

Kliegel, M., Martin, M., McDaniel, M. A., \& Einstein, G. O. (2001). Varying the importance of a prospective memory task: Differential effects across time- and event-based prospective memory. Memory, 9(1), 1-11. doi:10.1080/09658210042000003

Kliegel, M., Martin, M., McDaniel, M. A., \& Einstein, G. O. (2004). Importance effects on performance in event-based prospective memory tasks. Memory, 12(5), 553-561. doi:10.1080/ 09658210344000099

Kliegel, M., McDaniel, M. A., \& Einstein, G. O. (2000). Plan formation, retention, and execution in prospective memory: A new approach and age-related effects. Memory \& Cognition, 28(6), 1041-1049. doi:10.3758/Bf03209352

Kliegel, M., Martin, M., McDaniel, M. A., \& Einstein, G. O. (2002). Complex prospective memory and executive control of working 
memory: A process model. Psychologische Beiträge, 44(2), 303318.

Kuhlmann, B. G., \& Rummel, J. (2014). Context-specific prospectivememory processing: Evidence for flexible attention allocation adjustments after intention encoding. Memory \& Cognition, 42(6), 943-949. doi:10.3758/s13421-014-0405-2

Kvavilashvili, L., \& Ellis, J. (1996). Varieties of intentions: Some distinctions and classifications. In M. Brandimonte, G. O. Einstein, \& M. A. McDaniel (Eds.), Prospective memory: Theory and applications (pp. 23-52). Mahwah, NJ: Erlbaum.

Light, L. L. (1991). Memory and aging: Four hypotheses in search of data. Annual Review of Psychology, 42(1), 333-376.

Marsh, R. L., Cook, G. I., \& Hicks, J. L. (2006). Task interference from event-based intentions can be material specific. Memory \& Cognition, 34(8), 1636-1643.

Marsh, R. L., Hicks, J. L., \& Cook, G. I. (2005). On the relationship between effort toward an ongoing task and cue detection in eventbased prospective memory. Journal of Experimental Psychology: Learning, Memory, and Cognition, 31(1), 68-75. doi:10.1037/ 0278-7393.31.1.68

Marsh, R. L., Hicks, J. L., \& Watson, V. (2002). The dynamics of intention retrieval and coordination of action in event-based prospective memory. Journal of Experimental Psychology: Learning, Memory, and Cognition, 28(4), 652-659.

Maxwell, S. E., \& Delaney, H. D. (2004). Designing experiments and analyzing data: A model comparison perspective . Erlbaum: Mahwah.

Maylor, E. A. (1996). Age-related impairment in an event-based prospective-memory task. Psychology and Aging, 11(1), 74-78.

Maylor, E. A., Smith, G., Della Sala, S., \& Logie, R. H. (2002). Prospective and retrospective memory in normal aging and dementia: An experimental study. Memory \& Cognition, 30(6), 871-884.

McDaniel, M. A., \& Einstein, G. O. (2000). Strategic and automatic processes in prospective memory retrieval: A multiprocess framework. Applied Cognitive Psychology, 14, S127-S144. doi:10.1002/Acp.775

McDaniel, M. A., \& Einstein, G. O. (2007). Prospective memory: An overview and synthesis of an emerging field. Thousand Oaks: SAGE.

McDaniel, M. A., Einstein, G. O., \& Rendell, P. G. (2008). The puzzle of inconsistent age-related declines in prospective memory: A multiprocess explanation. In M. Kliegel, M. A. McDaniel, \& G. O. Einstein (Eds.), Prospective memory: Cognitive, neuroscience, developmental, and applied perspectives (pp. 141-160). New York, NY: Taylor \& Francis.

McDaniel, M. A., Einstein, G. O., Stout, A. C., \& Morgan, Z. (2003). Aging and maintaining intentions over delays: Do it or lose it. Psychology and Aging, 18(4), 823-835. doi:10.1037/0882-7974.18.4.823

McDaniel, M. A., Guynn, M. J., Einstein, G. O., \& Breneiser, J. (2004). Cuefocused and reflexive-associative processes in prospective memory retrieval. Journal of Experimental Psychology: Learning, Memory, and Cognition, 30(3), 605-614. doi:10.1037/0278-7393.30.3.605

McDaniel, M. A., \& Einstein, G. O. (2011). The Neuropsychology of Prospective Memory in Normal Aging: A Componential Approach. Neuropsychologia, 49(8), 2147-2155. doi:10.1016/j. neuropsychologia.2010.12.029.

Meier, B., \& Zimmermann, T. D. (2015). Loads and loads and loads: The influence of prospective load, retrospective load, and ongoing task load in prospective memory. Frontiers in Human Neuroscience, 9 , 322. doi:10.3389/fnhum.2015.00322

Mullet, H. G., Scullin, M. K., Hess, T. J., Scullin, R. B., Arnold, K. M., \& Einstein, G. O. (2013). Prospective memory and aging: evidence for preserved spontaneous retrieval with exact but not related cues. Psychology and Aging, 28(4), 910-922. doi:10.1037/a0034347

Neulinger, K., Oram, J., Tinson, H., O'Gorman, J., \& Shum, D. H. K. (2016). Prospective memory and frontal lobe function. Aging, Neuropsychology, and Cognition, 23(2), 171-183. doi:10.1080/ 13825585.2015.1069252
Niedzwienska, A., \& Barzykowski, K. (2012). The age prospective memory paradox within the same sample in time-based and event-based tasks. Aging, Neuropsychology, and Cognition, 19(1/2), 58-83. doi: 10.1080/13825585.2011.628374

Otani, H., Landau, J. D., Libkuman, T. M., St Louis, J. P., Kazen, J. K., \& Throne, G. W. (1997). Prospective memory and divided attention. Memory, 5(3), 343-360. doi:10.1080/741941393

Park, D. C., Hertzog, C., Kidder, D. P., Morrell, R. W., \& Mayhorn, C. B. (1997). Effect of age on event-based and time-based prospective memory. Psychology and Aging, 12(2), 314-327.

Pereira, A., Ellis, J., \& Freeman, J. (2012). Is prospective memory enhanced by cue-action semantic relatedness and enactment at encoding? Consciousness and Cognition, 21(3), 1257-1266. doi: 10.1016/j.concog.2012.04.012

Reese-Melancon, C. (2013). Age, focal processing, and monitoring in event-based prospective memory. Journal of Adult Development, 20(3), 151-157. doi:10.1007/s10804-013-9166-6

Rendell, P. G., McDaniel, M. A., Forbes, R. D., \& Einstein, G. O. (2007). Age-related effects in prospective memory are modulated by ongoing task complexity and relation to target cue. Aging Neuropsychology and Cognition, 14(3), 236-256. doi:10.1080/13825580600579186

Rose, N. S., Rendell, P. G., McDaniel, M. A., Aberle, I., \& Kliegel, M. (2010). Age and individual differences in prospective memory during a "virtual week": The roles of working memory, vigilance, task regularity, and cue focality. Psychology and Aging, 25(3), 595-605. doi: $10.1037 / \mathrm{a} 0019771$

Scullin, M. K., McDaniel, M. A., \& Einstein, G. O. (2010). Control of cost in prospective memory: Evidence for spontaneous retrieval processes. Journal of Experimental Psychology: Learning, Memory, and Cognition, 36(1), 190-203. doi:10.1037/a0017732

Scullin, M. K., McDaniel, M. A., \& Shelton, J. T. (2013). The Dynamic Multiprocess Framework: Evidence from prospective memory with contextual variability. Cognitive Psychology, 67(1/2), 55-71. doi:10. 1016/j.cogpsych.2013.07.001

Scullin, M. K., McDaniel, M. A., Shelton, J. T., \& Lee, J. H. (2010). Focal/nonfocal cue effects in prospective memory: monitoring difficulty or different retrieval processes? Journal of Experimental Psychology: Learning, Memory, and Cognition, 36(3), 736-749. doi: $10.1037 / \mathrm{a} 0018971$

Smith, R. E., \& Bayen, U. J. (2006). The source of adult age differences in event-based prospective memory: A multinomial modeling approach. Journal of Experimental Psychology: Learning, Memory, and Cognition, 32(3), 623-635. doi:10.1037/0278-7393.32.3.623

Smith, R. E., \& Hunt, R. R. (2014). Prospective memory in young and older adults: The effects of task importance and ongoing task load. Aging, Neuropsychology, and Cognition, 21(4), 411-431. doi:10. 1080/13825585.2013.827150

Vercambre, M. N., Cuvelier, H., Gayon, Y. A., Hardy-Leger, I., Berr, C., Trivalle, C., \& Clavel-Chapelon, F. (2010). Validation study of a French version of the modified telephone interview for cognitive status (F-TICS-m) in elderly women. International Journal of Geriatric Psychiatry, 25(11), 1142-1149. doi:10.1002/Gps.2447

Vergauwe, E., Barrouillet, P., \& Camos, V. (2010). Do mental processes share a domain-general resource? Psychological Science, 21(3), 384-390. doi:10.1177/0956797610361340

Verhaeghen, P., \& Salthouse, T. A. (1997). Meta-analyses of agecognition relations in adulthood: Estimates of linear and nonlinear age effects and structural models. Psychological Bulletin, 122(3), 231-249. doi:10.1037/0033-2909.122.3.231

Vogels, W. W., Dekker, M. R., Brouwer, W. H., \& de Jong, R. (2002). Age-related changes in event-related prospective memory performance: A comparison of four prospective memory tasks. Brain and Cognition, 49(3), 341-362.

Wechsler, D. (2011). Echelle d'intelligence de Wechsler pour adultes (WAIS-IV) - Quatrième édition. [Wechsler Adult Intelligence Scale (WAIS-IV) - Forth edition]. Montreuil: ECPA/Pearson. 
Welsh, K. A., Breitner, J. C. S., \& Magruderhabib, K. M. (1993). Detection of dementia in the elderly using telephone screening of cognitive status. Neuropsychiatry, Neuropsychology, and Behavioral Neurology, 6(2), 103-110.

West, R., \& Craik, F. I. (2001). Influences on the efficiency of prospective memory in younger and older adults. Psychology and Aging, 16(4), 682-696.
Zöllig, J., West, R., Martin, M., Altgassen, M., Lemke, U., \& Kliegel, M. (2007). Neural correlates of prospective memory across the lifespan. Neuropsychologia, 45(14), 3299-3314. doi:10.1016/J. Neuropsychologia.2007.06.010

Zuber, S., Kliegel, M., \& Ihle, A. (2016). An individual difference perspective on focal versus nonfocal prospective memory. Memory \& Cognition, 44(8), 1192-1203. doi:10.3758/s13421-016-0628-5 